\title{
SEMI-MAJOR AXIS KNOWLEDGE AND GPS ORBIT DETERMINATION
}

\section{J. Russell Carpenter" and Emil R. Schiesser ${ }^{\dagger}$}

In recent years spacecraft designers have increasingly sought to use onboard Global Positioning System receivers for orbit determination. The superb positioning accuracy of GPS has tended to focus more attention on the system's capability to determine the spacecraft's location at a particular epoch than on accurate orbit determination, per se. The determination of orbit plane orientation and orbit shape to acceptable levels is less challenging than the determination of orbital period or semi-major axis. It is necessary to address semi-major axis mission requirements and the GPS receiver capability for orbital maneuver targeting and other operations that require trajectory prediction. Failure to determine semi-major axis accurately can result in a solution that may not be usable for targeting the execution of orbit adjustment and rendezvous maneuvers. Simple formulas, charts, and rules of thumb relating position, velocity, and semi-major axis are useful in design and analysis of GPS receivers for near circular orbit operations, including rendezvous and formation flying missions. Space Shuttle flights of a number of different GPS receivers, including a mix of unfiltered and filtered solution data and Standard and Precise Positioning Service modes, have been accomplished. These results indicate that semi-major axis is often not determined very accurately, due to a poor velocity solution and a lack of proper filtering to provide good radial and speed error correlation.

- Aerospace Engineer, Guidance, Navigation, and Control Center, Code 572, NASA Goddard Space Flight Center, Greenbelt, MLZ077I.

$\uparrow \quad$ Specialist Engineering, The Boeing Company Space and Defense Systems - Houston Division, Houston, TX 77058. 


\section{INTRODUCTION}

For most people familiar with celestial mechanics, the Keplerian elements are among the most intuitive of the various sets used to describe orbital motion. However, in the modern era, orbit determination has mostly been accomplished using Cartesian coordinates. Cartesian parameters in recent years are also used in Global Positioning System (GPS) orbit determination. GPS receivers have the ability to determine accurate instantaneous position, and in some cases adequately accurate instantaneous velocity, but the determination of a state vector useful for state prediction, maneuver planning, etc., is another matter.

Orbital state determination and prediction accuracy depends on, among other factors, the error in force models used rather than on the size of the forces affecting spacecraft motion. For nearcircular orbits, the error in state prediction is due chiefly to initial orbital period or semi-major axis error, and imperfect force models used in prediction. The contributions from spacecraft venting, uncoupled attitude control thrusting, atmospheric drag, and solar radiation pressure force model errors are dependent on the type of vehicle, orbital characteristics, and the prediction interval. For some orbital applications in which gravitational field error is of prime interest, it is necessary to consider the effect of tides.

The accuracy with which orbital period can be determined depends on measurement type and geometry, and the error associated with force models used in the navigation process. Traditional satellite orbit determination uses measurements, such as range and Doppler, from ground tracking stations or tracking and data relay satellites. It is necessary to use data spread over relatively long arcs ( $3 / 4$ to several revolutions) from these sources to accurately determine the orbit since 
the measurements are not continuous and relate to only a portion of the state at a time. GPS provides continuous measurements that relate to all components of the state at a time so that, if desired, accurate local instantaneous position and velocity may be determined with a low order gravitational field model and a filter with a short memory. Such a solution might not provide accurate orbit state prediction since only a small portion of the orbit is measured. The accuracy may be improved through the use of a high-order gravitational field for both state determination and prediction, or as for traditional processes, a low order field but with a much longer filter memory.

Despite these complications, relatively simple means were developed, at least as far back as the 1960s, for the assessment of state prediction error due to initial state vector error and the effect of unmodeled forces. The intent of this paper is to expose long-standing back-of-the-envelope analyses of the orbital period error of an initial state vector, as reflected by semi-major axis error. The variation (osculation) in semi-major axis due to the non-spherical earth gravitational field is not relevant to the subject of the paper, assuming that an adequately accurate earth gravitational field model is used for the chosen navigation process.

\section{Coordination of Position and Velocity}

Coordinated position and velocity estimates are required to determine orbit orientation, shape and period or semi-major axis (SMA). The error in predicted along-track position depends on the error in orbital period or the error in SMA, as affected by the proper coordination of position and velocity knowledge. These errors in position and velocity can be considered to be knowl- 
edge errors in the current orbit, or maneuver execution errors created in trying to achieve an orbit change. Kepler discovered that for an ideal orbit, the square of the period is proportional to the cube of the semi-major axis. Letting $T_{p}$ denote the period, and $a$ denote the SMA, Kepler's law may be written'

$$
T_{p}=2 \pi \sqrt{\frac{a^{3}}{\mu}},
$$

where $\mu=G M$, the product of the gravitational constant, $G$, and the planetary mass, $M$. Eq. (1) may be used to establish a relationship ${ }^{2}$ between a variation in $\delta a$, the nominal SMA, and a variation in orbital period, $\delta T_{p}$ :

$$
\delta T_{p}=3 \pi \sqrt{\frac{a}{\mu}} \delta a .
$$

Based on the principle of conservation of energy, the sum of kinetic and potential energy, $-\mu /(2 a)$, is a constant ${ }^{1}$ in the absence of contact forces, such as drag, vents, or maneuvers. Thus, SMA knowledge is also an indication of knowledge of the orbit's energy. Letting $v$ denote velocity magnitude and $r$ position magnitude, the energy integral may be written

$$
\frac{1}{a}=\frac{2}{r}-\frac{v^{2}}{\mu},
$$

from which the following variational equation is derived:

$$
\frac{1}{a^{2}} \delta a=\frac{2}{r^{2}} \delta r+\frac{2 v}{\mu} \delta v .
$$

Eq. (3) is based on the assumption that the earth is spherical and the orbit is a circle or ellipse. For actual orbits, the $J_{2}$ oblateness term causes variations on the order of a few kilometers 
throughout a revolution compared to a spherical Earth orbit. Variation in Earth mass distribution beyond the $J_{2}$ Earth flattening results in additional but smaller departures from a conic orbit. Transformation from instantaneous "osculating" orbit elements to so-called mean element sets is often useful. However, this paper is primarily concerned with the analysis of variations in the osculating semi-major axis, which is derived from the instantaneous position and velocity, and variational analysis can be performed using Keplerian equations.

Many orbits of practical interest, such as low Earth orbits (LEO) and geosynchronous Earth orbits (GEO), are nearly circular. For a circular orbit, $a=r$, and from Eq. (3) $v=(\mu / r)^{1 / 2}$. With these assumptions, Eq. (4) simplifies to Eq. (5):

$$
\delta a=2 \delta r+\frac{2 r}{v} \delta v
$$

It is possible to have an error in radius and an error in speed but no error in SMA. The relationship between the radius and speed error for zero SMA error is shown in Eq. (6), which was obtained by setting $\delta a$ to zero in Eq. (5).

$$
\delta r=-\frac{r}{v} \delta v
$$

For LEO cases, the ratio $r / v$ is on the order of 1000 . Thus a one meter per second down-track velocity perturbation must be balanced by a -1000 meter radial position perturbation if SMA is to be maintained.

Position and velocity coordination affects flight-path angle in manner similar to that for the SMA. The flight-path angle, $\gamma$, is the complement of the angle between the velocity vector, $\mathbf{v}$ 
and the position vector, $r$, and can be visualized as the angle between the velocity vector and a plane perpendicular to the radius vector:

$$
r v \sin \gamma=\mathbf{r} \cdot \mathbf{v}
$$

The flight path angle is zero for a non-osculating circular orbit, which implies $\mathbf{r} \cdot \mathbf{v}=0$.

It is useful to consider an orthogonal set of perturbations in the radial, along-track, and crosstrack directions, $\delta r_{R}, \delta r_{l}, \delta r_{C}$, and their time derivatives, denoted by $\delta v_{R}, \delta v_{l}, \delta v_{C}$. Figure 1 illustrates the radial, along-track, and cross-track perturbations.

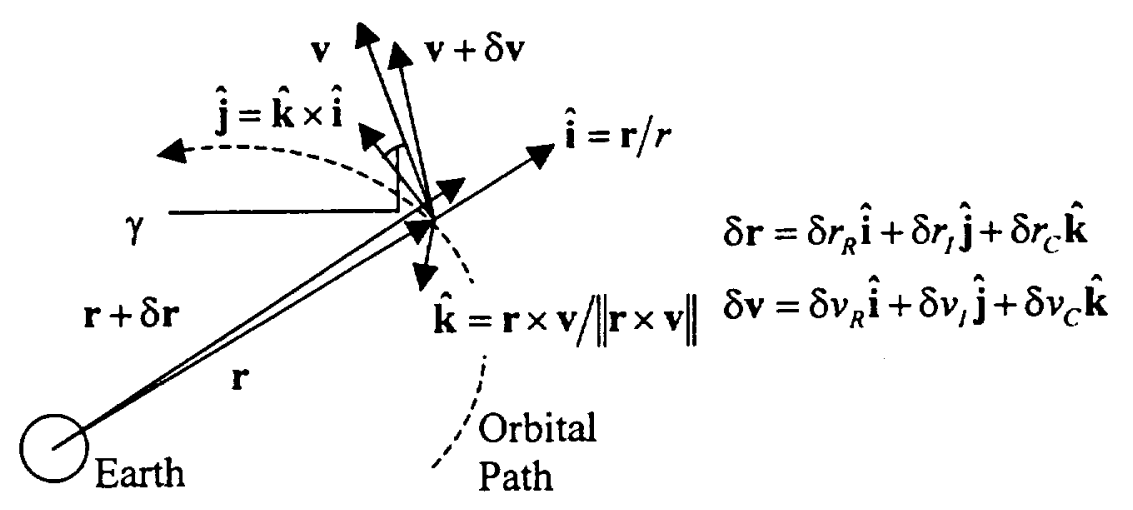

Figure 1: Radial, along-track, and cross-track perturbations.

In terms of the notation used above, $\delta r_{R} \approx \delta r$, and for near-circular orbits, velocity deviation is nearly all along-track, so that $\delta v_{I} \approx \delta v$. In terms of these perturbations, a relationship between deviations in along-track position, $\delta r_{1}$, and radial rate, $\delta v_{R}$, for zero flight path angle may be derived:

$$
\begin{aligned}
0 & =\mathbf{r} \cdot \mathbf{v} \\
& \approx[r, 0,0] \cdot\left[\delta v_{R}, \delta v_{l}, \delta v_{C}\right]+[0, v, 0] \cdot\left[\delta r_{R}, \delta r_{l}, \delta r_{C}\right] \\
& =r \delta v_{R}+v \delta r_{l}
\end{aligned}
$$


Eq. (8) reveals that if $\gamma$ is held to zero to preserve the nominally zero flight-path angle, then a change in along-track position, $\delta r_{l}$, must be compensated by a change in radial velocity:

$$
\delta s_{R}=-\frac{v}{r} \delta r_{l}
$$

The same rule-of-thumb ratio of approximately 1000 to 1 seen for radial and speed error correlation for zero SMA error shows up again in Eq. (9) for the relationship between along-track position and radial rate for zero flight path angle deviation. A one meter per second perturbation to radial velocity must be balanced by a -1000 meter change in down-track position, if near-zero flight-path angle is to be maintained.

\section{Effects of Poor Semi-Major Axis Knowledge}

Along-track error growth. A semi-major axis error reflects a period error (Eq. (2)) and the satellite will complete more or less than one actual revolution during its nominal period than predicted. For near-circular orbits, the resulting along-track error is

$$
\delta r_{l}=-v\left(t / T_{p}\right) \delta T_{p}
$$

where the negative sign convention recognizes that if the actual period is less than nominal, the satellite ends up farther along in its orbit than nominal, which is taken to be a positive alongtrack error. Setting $t=T_{p}$ in Eq. (10) gives the along-track error per revolution:

$$
\delta r_{1}=-v \delta T_{p},
$$

Substituting Eq. (2) into Eq. (11), and assuming the velocity to be the circular orbit velocity, results in Eq. (12):

$$
\delta r_{l}=-3 \pi \delta a
$$


which leads to a common rule of thumb for along-track error growth per orbit revolution, i.e. the along track error per revolution is equal to minus ten times the semi-major axis error ${ }^{2}$.

Manewver execution errors. The performance of a proper orbital maneuver is affected by targeting error, which is usually dominated by local SMA error, and imperfect execution of the targeted maneuver. Maneuver execution error may be due to inaccurate measurement of the velocity change or the duration of the maneuver, depending on execution approach, as well as improper maneuver attitude and an imperfect thrust "tailoff" model. In the case of an instantaneous or short maneuver, there is no appreciable change to the position error, and any along-track velocity execution error will not be balanced by a corresponding radial position error, and will result in a semi-major axis error per Eq. (6).

Covariance matrix propagation. A less familiar example occurs in the problem of initializing the covariance matrix for a sequential orbit determination filter that uses Cartesian coordinates. Sequential state determination provides a current running estimate, but has the feature that only a portion of the state is typically observable when using the measurement set available at current time. The filter retains the history of past measurement processing as an estimate of the uncertainty of the state in the form of a variance/co-variance matrix that must be initialized. The initial covariance matrix should reflect the uncertainty in the initial state vector. The state and its uncertainty (in the form of the covariance matrix) have to be propagated for some time before the first measurement becomes available. As a result, the initial covariance matrix must reflect the proper uncertainty in SMA and flight path angle. Eqs. (6) and (9) may be used to establish radial, speed, radial-rate and along-track variances and associated co-variances. 
Figure 2 illustrates the effect of the initial covariance matrix on propagation. The matrix shown has units of $\mathrm{m}$ and $\mathrm{m} / \mathrm{s}$ and the rows and columns are $r_{R}, r_{l}, r_{c}, v_{R}, v_{l}$, and $v_{c}$. The initial covariance shown on the plot was propagated without process noise, in a circular low Earth orbit whose period is 5828.5 seconds to produce the figure. The initial value of the SMA uncertainty (computed using Eq. (16) shown in the next section) is given at the top of each plot. The upper subplot shows overall error growth when the error in radius matches the error in speed per the rule of thumb (1000 m radial error per $-1 \mathrm{~m} / \mathrm{s}$ speed error). The lower subplot shows error

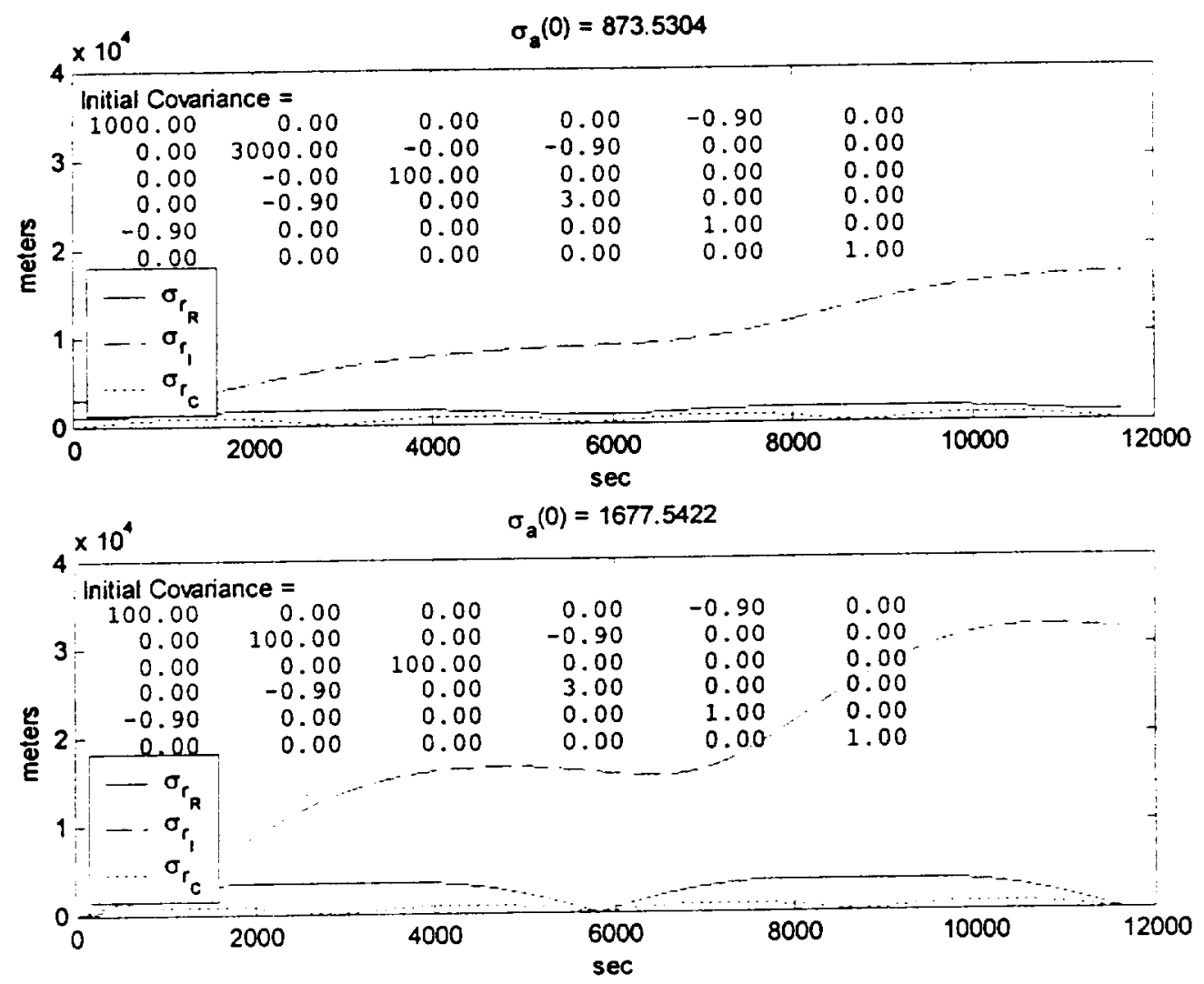

Figure 2: Comparison of covariance propagation with matched and unmatched SMA and flight-path angle relationships 
growth for unmatched errors one might expect trom some GPS point solutions. The $100 \mathrm{~m}$ radial error (smaller than the $1000 \mathrm{~m}$ radial error in the upper matrix) cannot compensate for the $1 \mathrm{~m} / \mathrm{s}$ speed error and results in along-track error growth that is about double that of the matched case, shown in the upper subplot. Either the $1 \mathrm{~m} / \mathrm{s}$ error must be reduced to $0.1 \mathrm{~m} / \mathrm{s}$ or the radial error must be increased to $1000 \mathrm{~m}$ to achieve reduced along-track error growth.

\section{SIMPLE FORMULAS FOR SEMI-MAJOR AXIS UNCERTAINTY}

This section illustrates some simple formulas and charts that relate the statistical variance of the Cartesian states to the semi-major variance. Modification of these formulas for relative navigation applications is also discussed.

\section{Absolute State Semi-major Axis}

For a Cartesian state vector $\mathbf{x}=\left[\mathbf{r}^{\top}, \mathbf{v}^{\top}\right]$, a first order approximation that relates changes in the state away from a nominal value (denoted by the subscript $o$ ) to changes in the SMA is given by

$$
a-\left.a_{o} \approx \frac{\partial a}{\partial \mathbf{x}}\right|_{\mathbf{x}=\mathbf{x}_{o}}\left(\mathbf{x}-\mathbf{x}_{o}\right)
$$

For

$$
A\left(\mathbf{x}_{o}\right)=\left.\frac{\partial a}{\partial \mathbf{x}}\right|_{\mathbf{x}=\mathbf{x}_{o}}=\left.2 a^{2}\left[\frac{\mathbf{r}^{\top}}{r^{3}}, \frac{\mathbf{v}^{\top}}{\mu}\right]\right|_{\mathbf{x}=\mathbf{x}_{o}},
$$

where the partial derivative has been evaluated assuming a two-body potential, the error variance of the SMA in terms of the state error covariance is 


$$
\begin{aligned}
& \mathrm{E}\left[\left(a-a_{0}\right)^{2}\right]=\mathrm{E}\left[A\left(\mathbf{x}_{0}\right)\left(\mathbf{x}-\mathbf{x}_{0}\right)\left(\mathbf{x}-\mathbf{x}_{0}\right)^{\top} A\left(\mathbf{x}_{0}\right)^{\top}\right] \\
& \sigma_{a}^{2}\left(\mathbf{x}_{u}\right)=A\left(\mathbf{x}_{o}\right) P_{\mathrm{x}} A\left(\mathbf{x}_{o}\right)^{\top}
\end{aligned}
$$

In deriving Eq. (15), Eqs. (13) and (14) have been used, the symbol "E" denotes the expectation operator, and $P_{x}$ is the state error covariance matrix. The following simplification of Eq. (15), valid for near-circular orbits may be found in Reference 2:

$$
\sigma_{a}=2 \sqrt{\sigma_{r_{R}}^{2}+2\left(\frac{T_{p}}{2 \pi}\right) \rho_{r_{R} v_{l}} \sigma_{r_{R}} \sigma_{v_{t}}+\left(\frac{T_{p}}{2 \pi}\right)^{2} \sigma_{v_{t}}^{2}} .
$$

This equation may also be derived from Eq. (5), using Eqs. 2 and 3.

In Eq. (16), $\rho_{r_{k} v_{f}}$ denotes the correlation coefficient between radial position error and in-track velocity error, and $\sigma_{r_{n}}$ and $\sigma_{v_{t}}$ denote the radial position error and in-track velocity error standard deviations, respectively.

Eq. (16) may be used as a design and analysis aid, especially in graphical form. Also, either Eq. (15) or Eq. (16) is useful as a "figure of merit" for orbit determination filters. Figure 3 shows one means of graphically representing Eq. (16) for LEO scenarios. Figure 3 depicts several families of relationships among the principal contributors to SMA uncertainty for a LEO mission. Each family of curves illustrates a subset of the range of radial position and along-track velocity errors that generate the same SMA error, from 0.1 meter to 1000 meters.

Along the main diagonal of the chart, the families split based on the value of the correlation coefficient. The correlation coefficient captures the deterministic relationship implied among the ra- 
dial position and down-track velocity errors by Eq. (6). To the extent that these errors are properly coordinated, a given level of knowledge about either one implies some degree of knowledge about the other. The coefficient is negative because of the negative relationship stated by Eq. (6). The region of the plot in which the families split corresponds to a "sweet spot" of proper coordination among position and velocity.

At one extreme, radial position and down-track velocity errors are completely uncorrelated $(\rho=0)$, and the SMA error is bounded below by the lower of its corresponding radial position and speed errors. For example, no matter how good position knowledge gets, SMA knowledge will be no better than about 100 meters if speed knowledge cannot be improved beyond about 0.05 meters per second. However, if radial position and speed errors are properly coordinated (via Eq. (6)), the SMA knowledge can be dramatically improved, because a corresponding increase in the correlation among radial position and down-track velocity errors is possible. For example, using the "1000 to 1" rule of Eq. (6), a radial position error of 100 meters should correspond to a down-track velocity error of about -.1 meters per second. As Figure 3 shows, this combination could correspond to an SMA error of about 100 meters if the correlation coefficient is -.9 , but would only correspond to an SMA error of just under 300 meters if the correlation coefficient is near zero. A coefficient near the value one would expect if Eq. (6) were nearly exactly satisfied $(\sim-0.99)$ would give an SMA error on the order of 30 meters. In the limit, the coefficient reaches -1 , the horizontal and vertical branches on the plot disappear, the random variables become simple deterministic variables, and the linear relationship of Eq. (6) is depicted as a straight line from lower left to upper right. 


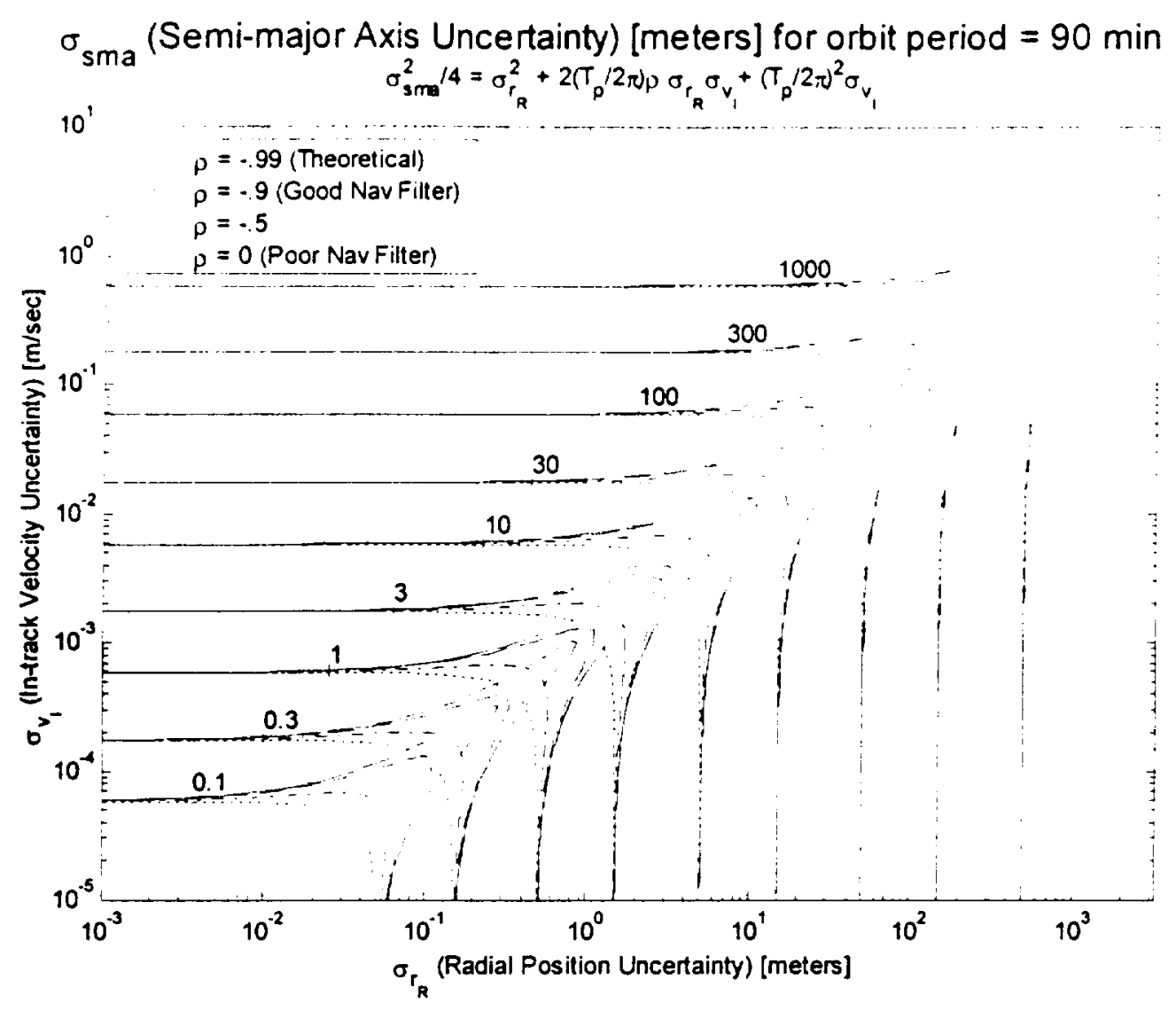

Figure 3: Semi-major axis uncertainty relationships for a typical low Earth orbit.

The two rules-of-thumb of Eqs. (6) and (9) may be used to specify the in-plane diagonal elements of the initial covariance. Eq. (6) may be used to specify the partition of the covariance corresponding to along-track position and radial velocity errors that are consistent with the SMA error of the initial state. For near-circular orbits, the remainder of the in-plane covariance partition may be specified using Eq. (9) to specify radial velocity and down-track position errors that are consistent with the approximately zero flight path angle of the initial state. When using both rules to specify an initial covariance, an appropriate selection of the correlation coefficient should be made, as discussed above. If Eqs. (6) and (9) have been used, a reasonably conservative choice of correlation coefficient is -.9 . 


\section{Relative State Semi-major Axis}

Relative SMA, i.e. the difference in SMA between pairs of vehicles, is of interest for rendezvous and formation flying missions. During rendezvous, a "chaser" will execute maneuvers to approach target spacecraft. Denoting the chaser SMA by $a_{c}$, and the target SMA by $a_{t}$, the relative SMA is

$$
a_{r e l}=a_{c}-a_{1}
$$

Since $a_{c}$ is a function of the chaser state $\mathbf{x}_{c}$ and $a_{t}$ is a function of the target state $\mathbf{x}_{t}$, in deriving the uncertainty of the relative SMA it is useful to define a state consisting of both the chaser and target states: $\mathbf{x}^{\top}=\left[\mathbf{x}_{c}^{\top} \mathbf{x}_{t}^{\top}\right]$. Then, as in Eq. (13), a perturbation in the nominal relative SMA may be approximated by

$$
a_{r e t}-\left.a_{r e l_{o}} \approx \frac{\partial a_{r e l}}{\partial \mathbf{x}}\right|_{\mathbf{x}=\mathbf{x}_{0}}\left(\mathbf{x}-\mathbf{x}_{0}\right)
$$

Using the notation of Eq. (14), the partial derivative is evaluated as follows:

$$
\begin{aligned}
\left.\frac{\partial a_{r e t}}{\partial \mathbf{x}}\right|_{\mathbf{x = \mathbf { x } _ { o }}} & =\left.\left[2 a_{c}^{2}\left[\frac{\mathbf{r}_{c}^{\top}}{r_{c}^{3}}, \frac{\mathbf{v}_{c}^{\top}}{\mu}\right],-2 a_{1}^{2}\left[\frac{\mathbf{r}_{t}^{\top}}{r_{t}^{3}}, \frac{\mathbf{v}_{t}^{\top}}{\mu}\right]\right]\right|_{\mathbf{x}=\mathbf{x}_{o}} \\
A\left(\mathbf{x}_{o}\right) & =\left[A_{c}\left(\mathbf{x}_{o}\right),-A_{t}\left(\mathbf{x}_{o}\right)\right]
\end{aligned}
$$

The covariance of the chaser/target state, $P_{x}$, may be partitioned into chaser-only and target-only covariances $\left(P_{c}\right.$ and $\left.P_{t}\right)$, and their cross-covariance matrices $\left(P_{c t}\right)$ :

$$
P_{x}=\left[\begin{array}{cc}
P_{c} & P_{c t} \\
P_{c t}^{\top} & P_{1}
\end{array}\right]
$$

Using Eqs. (19) and (20) in Eq. (15) results in the following: 


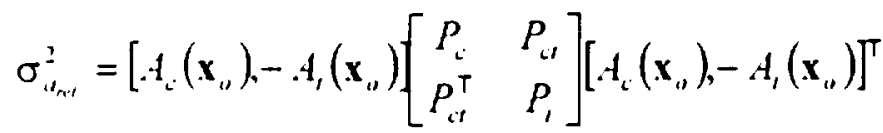

$$
\begin{aligned}
& =A_{c} P_{c} A_{c}^{\top}+A_{1} P_{1} A_{1}^{\top}-A_{c} P_{c 1} A_{1}^{\top}-\left(A_{c} P_{c} A_{1}^{\top}\right)^{\top}
\end{aligned}
$$

\section{FLIGHT PERFORMANCE SURVEY}

A number of different GPS receivers have been flown on Space Shuttle Orbiter flights, including a mix of GPS units, some which filtered measurement data and some which extracted the state deterministically from the data. Some of the GPS units operated in Standard Positioning Service and others in Precise Positioning Service modes. Both absolute state and relative state performance have been evaluated. Particular interest has been given to the semi-major axis performance of the GPS receivers and any associated filtering algorithms, to determine if the GPS has the potential to replace or augment rendezvous navigation sensors.

\section{Absolute State Flight Performance}

Twenty-one sets of absolute-state flight data results are shown in Table 1, and in Figure 4, the flight data are overlaid on a copy of Figure 3. The data used in constructing these tables was extracted from Refs. 3-6, and from various internal documents. In some cases, either velocity or SMA data were not available. In general, the flight data indicate that semi-major axis is often not determined very accurately, typically due to a poor velocity solution and/or a lack of proper filtering to provide good radial and speed error correlation. Note that the flight data are all from LEO missions, and have periods varying by a few minutes from the nominal period of 90 minutes used to construct the figure.

Nearly all the flight data lie above the diagonal region in Figure 4 previously described as the 
"sweet spot" for proper coordination of position and velocity. This indicates that, for these cases, GPS-derived velocity is not as good as GPS-derived position, so that GPS is not able to achieve good SMA accuracy. Cases $J$ and $B$ happen to lie in the "sweet spot," but Table 1 reveals that their SMA accuracy corresponds to a near-zero correlation coefficient. Thus, these cases are not taking advantage of the potential improvement in SMA to the order of 10-30 m they could otherwise achieve.

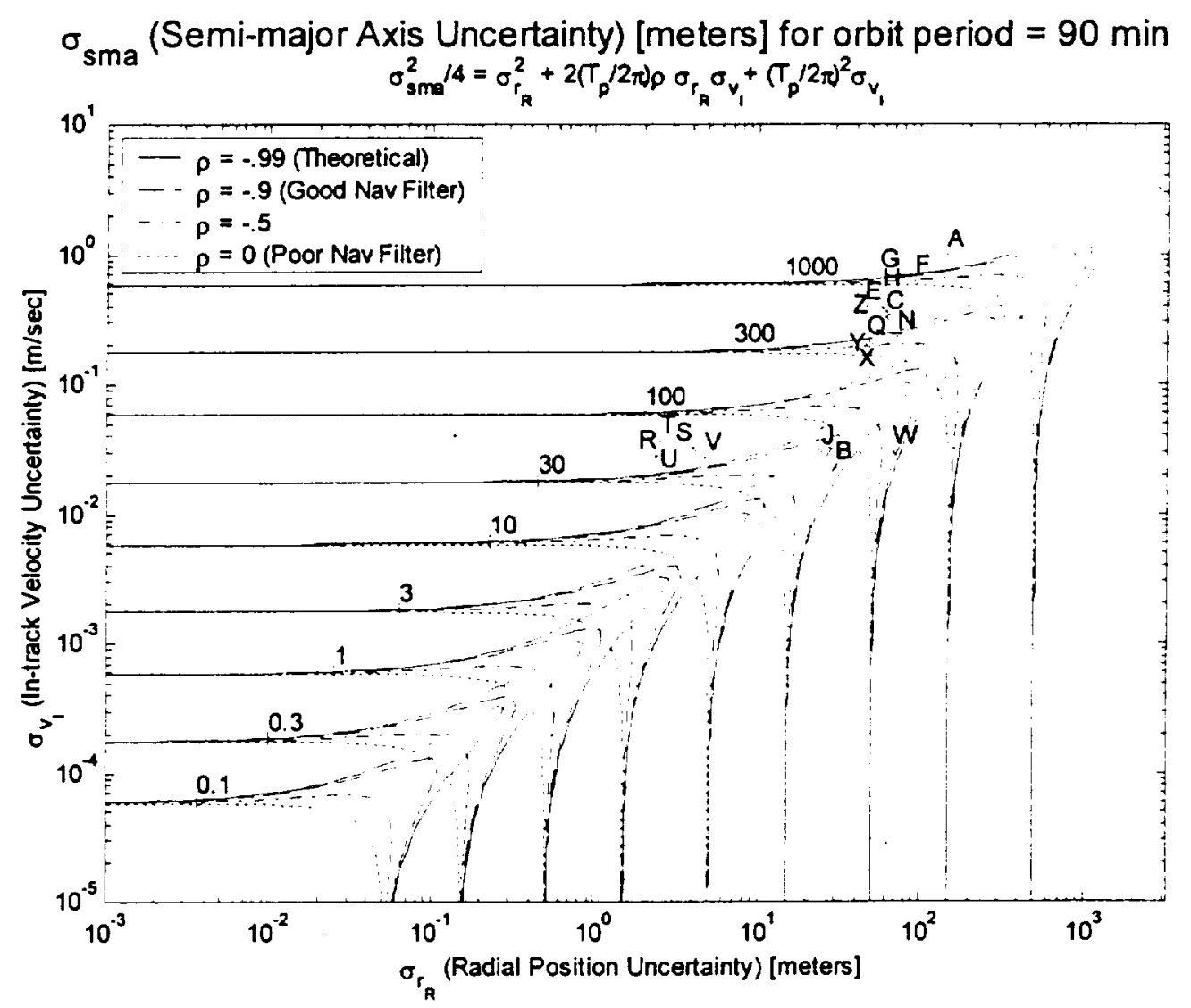

Figure 4: Semi-major axis uncertainty flight data results overlaid on SMA uncer-

Cases $W, X, Y$, and $Z$ are point solutions produced onboard TOPEX satellite, during which Selective Availability (SA) and Anti-Spoofing (AS) were in various states of activation, as Table 1 
describes. Although TOPEX's 112 minute period is approximately $25 \%$ longer than the nominal period used to produce Figures 3 and 4, its SMA is only 13\% larger, an error which is not significant on the logarithmic scale the figures use. With this caveat in mind, it appears that the SA/AS-free case, Case $W$, actually has a velocity that is good enough to produce an SMA error on the order of $30 \mathrm{~m}$. However, its position solutions are not quite accurate enough, nor perhaps is its correlation of position and velocity, as Table 1 indicates its SMA error due be over $100 \mathrm{~m}$.

To establish performance, flight data were compared to best estimated trajectories (BETs), generated with various procedures. Most of the BET procedures have been verified to be accurate to about 30 meters RMS in position and a few hundredths of a meter per second RMS in velocity? The Post-flight Attitude and Trajectory History (PATH) BET is probably less accurate in position, but more accurate in velocity, than the Wide Area Differential GPS (WADGPS) BET. (The PATH accuracy is specified to be on the order of 200 meters in position and $0.2 \mathrm{~m} / \mathrm{s}$ in velocity RMS in the worst axis, but it is generally believed to be much better.) In some cases, filtered position data from an independent, PPS-capable GPS receiver was used as the BET. These BETs are believed to be slightly more accurate in position than the WADGPS BET is, and comparable to the PATH BET in velocity. The SLR+DORIS BETs used for the TOPEX data are accurate to a few $\mathrm{cm}$ in position, and a few tenths of a $\mathrm{mm} / \mathrm{sec}$ in velocity. 


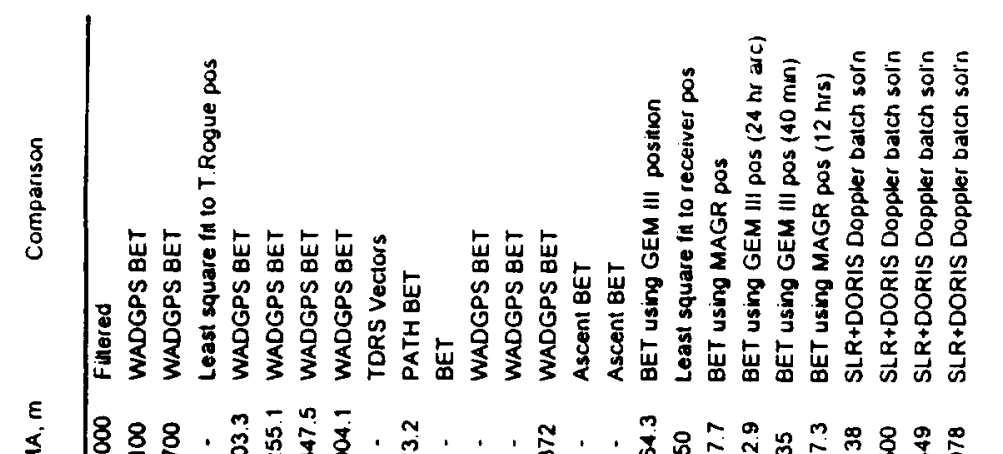

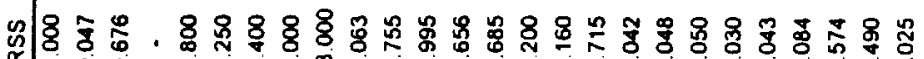

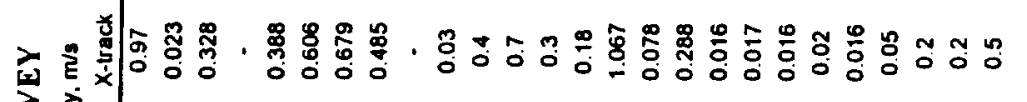

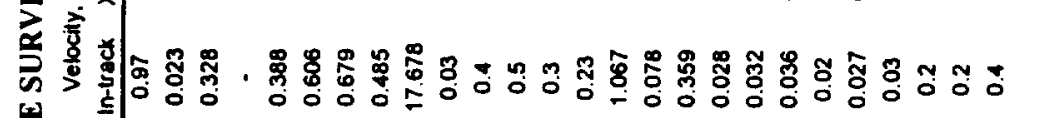

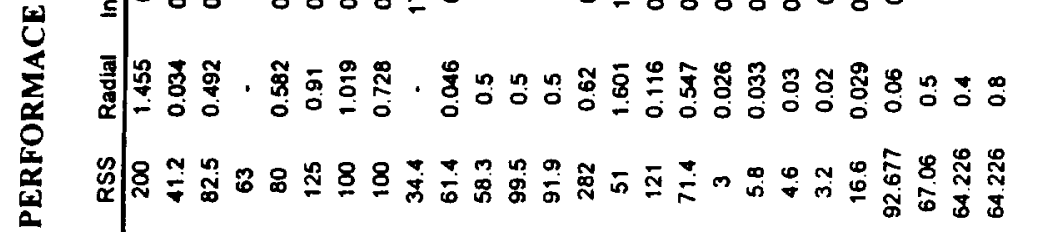

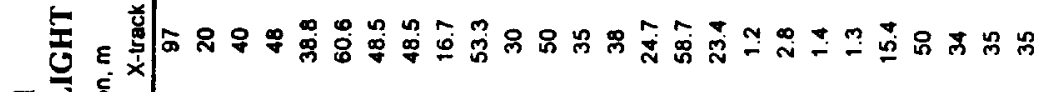

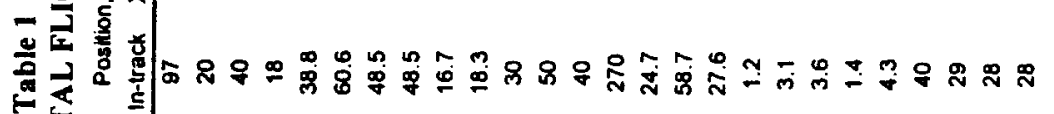

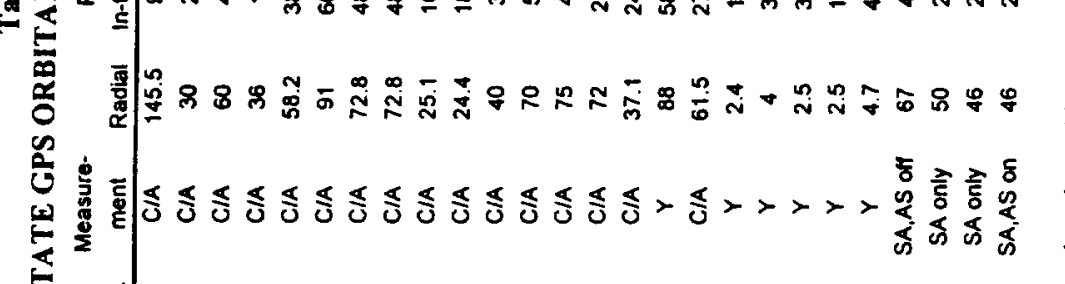

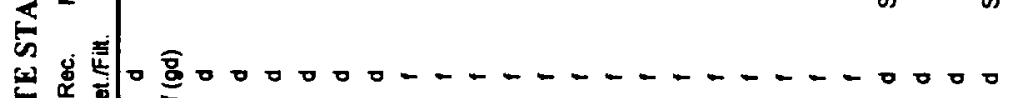

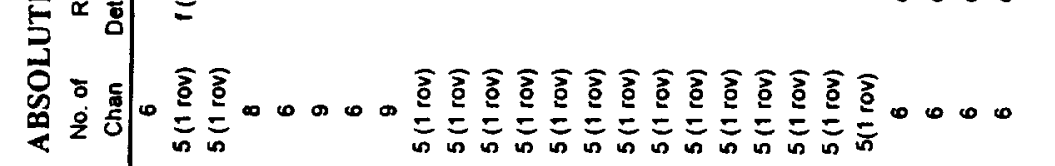

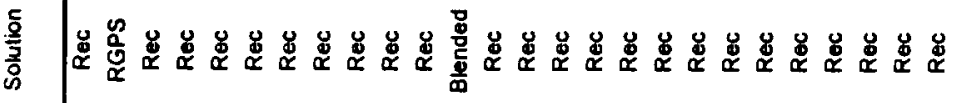

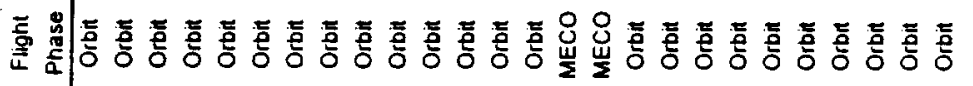

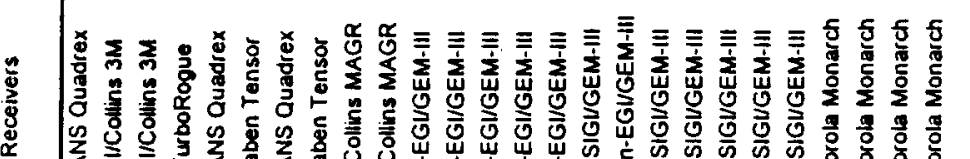
WMIM!Mm!m!III

ระ

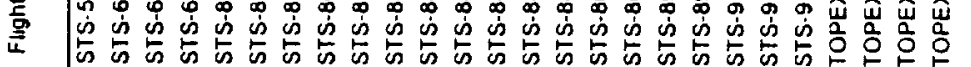

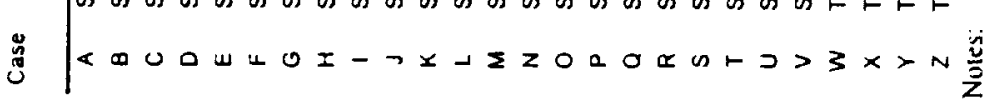

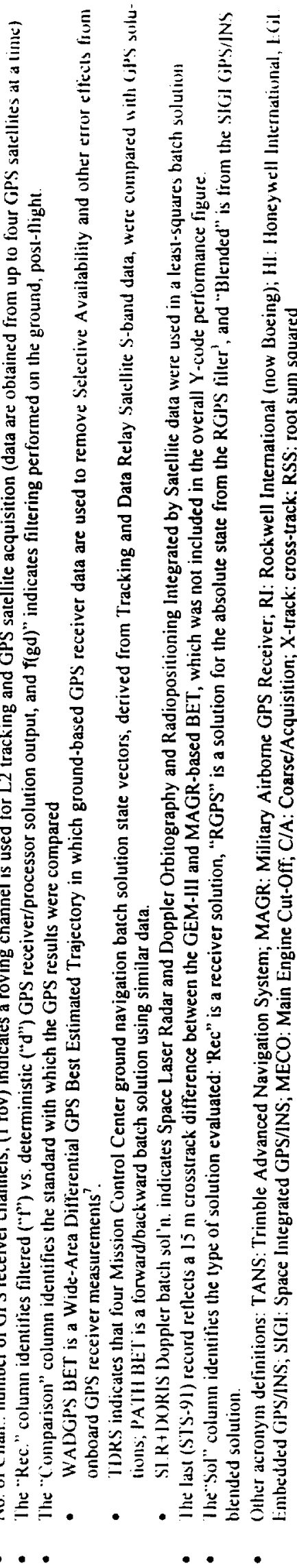




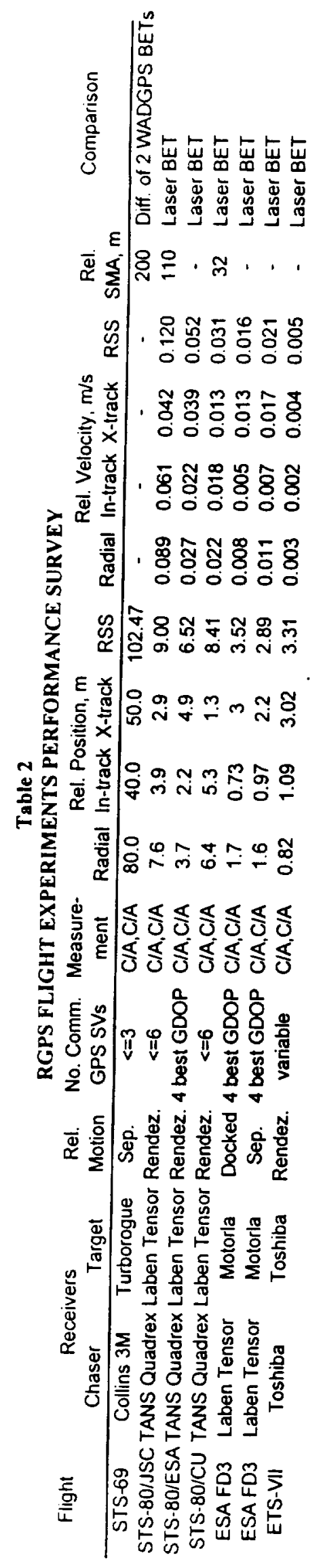




\section{Relative State Flight Performance}

NASA has jointly performed two relative GPS (RGPS) navigation flight experiments with the European Space Agency (ESA) ${ }^{3-6}$. During these experiments, a near-realtime relative GPS Kalman filter processed pseudorange data from GPS receivers aboard the Space Shuttle and a free flying satellite deployed and retrieved by the Shuttle. This filter was designed to estimate common biases affecting GPS receivers aboard two co-orbiting spacecraft tracking the same GPS satellites, and was expected to approach pseudorange (not carrier phase) differential type performance when measurements from at least four common satellites were processed. Recently, both ESA and the National Space Development Agency of Japan have performed further relative GPS experiments ${ }^{8,9}$. STS-80 data have also been processed at the University of Colorado at Boulder $(\mathrm{CU})^{10}$. Table 2 summarizes relative state performance from the various RGPS experiments.

The RGPS results may be compared with Table 3, which shows typical performance of the existing Shuttle rendezvous navigation system. The existing system consists of a rendezvous radar

\section{Table 3}

\section{PERFORMANCE OF EXISTING SHUTTLE RENDEZVOUS SYSTEM}

\begin{tabular}{|c|c|c|c|c|c|c|c|c|}
\hline \multicolumn{4}{|c|}{ Position (m) } & \multicolumn{4}{|c|}{ Velocity $(\mathrm{m} / \mathrm{s})$} & SI \\
\hline $\operatorname{Radi}$ & In-track & X-track & RSS & Radial & In-track & X-track & RSS & \\
\hline 15.1 & 3.6 & 1.7 & 15.6 & 0.023 & 0.015 & 0.008 & 0.029 & 49.4 \\
\hline
\end{tabular}

that provides range, range-rate, azimuth and elevation measurements to an extended Kalman filter that also utilizes acceleration data from an inertial measurement unit. The data were derived by comparison of the downlinked Shuttle relative navigation data to a laser-based BET produced after the flight. Although the relative GPS position accuracy shown in Table 2 is somewhat bet- 
ter than the relative position accuracy of the Shuttle's existing system shown in Table 3 , the GPS-based relative SMLA is more than twice as inaccurate. The performance difference is primarily due to poorer relative velocity estimates from the RGPS filter, but also due to poorer correlation among position and velocity errors.

Another approach to GPS relative navigation is to directly difference the solutions from two GPS

Table 4

STATE VECTOR DIFFERENCING PERFORMANCE FROM STS-80

\begin{tabular}{|c|c|c|c|c|c|c|c|c|c|c|}
\hline Sol & Measure- & \multicolumn{4}{|c|}{ Position (m) } & \multicolumn{3}{|c|}{ Velocity (m/s) } & SMA (m) \\
\hline DevFilt & ment & Radial & In-track & X-track & RSS & Radial & In-track & X-track & RSS & \\
\hline \hline d,d & C/A, C/A & 138.0 & 51.7 & 57.0 & 158.1 & 1.699 & 0.949 & 0.550 & 2.022 & 1706.0 \\
\hline
\end{tabular}

receivers, rather than process the measurement data from both receivers in a single Kalman filter as described above. Such an approach is obviously much simpler, but does not necessarily remove the common error sources. As Table 4 shows, the state vector differencing performance data from STS-80 show significantly worse performance than the filtered results Table 2 presents.

However, if the receivers can be operated under the Precise Positioning Service (PPS), with dualfrequency $\mathrm{P} / \mathrm{Y}$-code measurements, most errors larger than a few meters are removed. Under such circumstances, it could be feasible to perform GPS relative navigation using state vector differencing. Absolute state flight performance was used to predict relative state accuracy for a variety of GPS receiver combinations with deterministic vs. filtered solutions based on C/A and $\mathrm{P} / \mathrm{Y}$-code measurements (Table 5). The values in Table 5 were obtained by computing the root sum squares of the overall absolute state performance summarized at the bottom of Table 1 , as- 
Table 5

STATE VECTOR DIFFERENCING PERFORMANCE SURVEY

\begin{tabular}{|c|c|c|c|c|c|c|c|c|c|c|}
\hline \multirow{2}{*}{$\begin{array}{c}\text { Sol } \\
\text { Devfilt } \\
\end{array}$} & \multirow{2}{*}{$\begin{array}{c}\text { Measure- } \\
\text { ment }\end{array}$} & \multicolumn{4}{|c|}{ Position (m) } & \multicolumn{4}{|c|}{ Velocity $(\mathrm{m} / \mathrm{s})$} & \multirow[t]{2}{*}{$\overline{S M A}(m)$} \\
\hline & & Radial & In-track & $x$-track & RSS & Radial & In-track & $x$-track & RSS & \\
\hline$d, d$ & $C / A, C / A$ & 92.1 & 60.0 & 67.0 & 128.7 & 1.055 & 0.703 & 0.703 & 1.450 & 1445.3 \\
\hline$d, f$ & $C / A, C / A$ & 83.7 & 52.2 & 59.1 & 115.0 & 0.872 & 0.582 & 0.589 & 1.203 & 1076.0 \\
\hline d $f$ & $\mathrm{C} / \mathrm{A}, \mathrm{Y}$ & 652 & 42.5 & 47.5 & 91.2 & 0.746 & 0.498 & 0.498 & 1.026 & 1023.3 \\
\hline$f, f$ & $C / A, C / A$ & 743 & 43.0 & 49.8 & 993 & 0.639 & $0.429^{\circ}$ & 0.448 & 0.891 & 475.9 \\
\hline$f, f$ & $C / A, Y$ & 52.7 & 30.6 & 35.3 & 70.4 & 0.453 & 0.305 & 0.317 & 0.631 & 340.3 \\
\hline$f, f$ & $Y, Y$ & 4.6 & 3.8 & 3.0 & 6.7 & 0.039 & 0.041 & 0.024 & 0.061 & 71.5 \\
\hline
\end{tabular}

suming that the errors in the two receivers are uncorrelated (i.e. $P_{c t}=0$ ). The results for absolute states computed deterministically by the receivers, and absolute states resulting from onboard orbit determination filters are denoted " $\mathrm{d}$ " and "f," respectively, in the first column. The assumption of uncorrelated errors is conservative, corresponding to the case in which the receivers are tracking zero common satellites. However, only the case in which all satellites tracked are common will reflect substantially better accuracy.

Although filtering the data helps significantly, state vector differencing, even if one of the two receivers is P/Y-code capable, is not competitive with either the RGPS filtering approach or the existing Shuttle rendezvous system capabilities (viz. Tables 2 and 3). Relative state accuracy, obtained by differencing two absolute states from GPS receivers which filter P/Y-code data, (shown in the last row of Table 5) was more accurate than RGPS filtered C/A code performance (Table 2), and comparable to existing Shuttle rendezvous performance (Table 3). There are no known flight results from an RGPS-type filter using $\mathrm{P} / \mathrm{Y}$-code measurements.

None of the flight data results presented utilize carrier phase data, which is commonly used in static surveying applications to achieve centimeter-level relative positioning. Carrier phase 
measurements are analogous to a very accurate pseudo-range measurement, if the measurement is obtained as an accumulation of phase, or equivalently range, change. Based on Figure 3 , it would appear that even if position were known to better than I centimeter, SMA knowledge could not be improved over existing levels of performance unless both velocity knowledge were obtained to the 0.1 to $1 \mathrm{~mm} / \mathrm{sec}$ level, and proper correlation between position and velocity errors were achieved.

\section{CONCLUSION}

Semi-major axis accuracy affects orbital targeting and other functions dependent on state propagation and prediction. On-orbit GPS absolute and relative SMA Shuttle flight performance results achieved to date, though promising, have often been less than desirable and at worst inadequate. The lack of good semi-major axis knowledge is primarily due to a poor velocity solution and a lack of proper filtering to provide good radial and speed error correlation. Use of carrier phase data does not appear likely to improve this situation, unless it is accompanied by proper filtering that is tuned to take advantage of orbital dynamics. Such a filter would create the necessary state correlations that accurate SMA knowledge implies. Several simple equations, rules of thumb, and charts concerning the semi-major axis used for back-of-the envelope orbit determination analysis have been presented to support future orbital navigation performance analysis. It is hoped that this work will stimulate innovations that will lead to better orbital GPS orbit determination performance.

\section{ACKNOWLEDGEMENTS}

Cary Semar of The Boeing Company Space and Defense Systems - Houston Division and the 
staff and support contractors of the Flight Design and Dynamics Division of NASA Johnson Space Center provided much of the data from Shuttle tlights of the Honeywell EGI and SIGI and the Litton EGI. Lou Zyla of The Boeing Company Space and Defense Systems - Houston Division provided the data from the STS-51 Shuttle GPS experiment. George Davis of Orbital Sciences Corporation provided the TOPEX data.

\section{REFERENCES}

1. R. Bate, D. Mueller, and J. White, Fundamentals of Astrodynamics, Dover, New York, 1971.

2. W. Lear, "Orbital Elements Including the $J_{2}$ Harmonic," Report No. 86-FM-18/JSC-22213, Rev. 1, Mission Planning and Analysis Division, NASA Johnson Space Center, 9/87.

3. Y. Park, et al., "Flight Test Results from Realtime Relative GPS Flight Experiment on STS-69," Space Flight Mechanics 1995.

4. C. Schroeder, B. Schutz, and P.A.M. Abusali, "STS-69 Relative Positioning GPS Experiment," Space Flight Mechanics 1995.

5. G. Moreau and H. Marcille, "FDI Post-Flight Analysis and Evaluation Report," Matra Marconi Space France Report No. ARPK-RP-SYS-3744-MMT, 11/8/97.

6. E. Schiesser, et al. "Results of STS-80 Relative GPS Navigation Flight Experiment," Space Flight Mechanics 1998.

7. M. Lisano and R. Carpenter, "High-Accuracy Space Shuttle Reference Trajectories for the STS-77 GPS Attitude and Navigation Experiment (GANE)," Space Flight Mechanics 1997.

8. G. Moreau and H. Marcille, "RGPS Post-flight Analysis of ARP-K Flight Demonstrations," Proceedings of the ION GPS-98, Nashville, TN, September 1998.

9. I. Kawano, et al., "Result and Evaluation of Autonomous Rendezvous Docking Experiment of ETS-VII," Proceedings of the AIAA GNC Conference, Portland, OR, August, 1999.

10. D. Highsmith and P. Axelrad, "Relative State Estimation Using GPS Flight Data from Co-orbiting Spacecraft," Proceedings of the ION GPS-99, Nashville, TN, September 1999. 